\title{
Expression of aquaporin-1 in endometrial blood vessels in menorrhagia
}

\author{
MIRIAM MINTS $^{1}$, ANNA HILDENBRAND ${ }^{2}$, LUTHER PARAMESWARAN GRACE LALITKUMAR ${ }^{2}$, \\ SONIA ANDERSSON ${ }^{1}$, SÖREN NIELSEN ${ }^{3}$, KRISTINA GEMZELL-DANIELSSON $^{2}$ \\ and ANNELI STAVREUS-EVERS ${ }^{1}$
}

\begin{abstract}
${ }^{1}$ Department of Clinical Science, Intervention and Technology, Division of Obstetrics and Gynecology, Karolinska University Hospital Huddinge, Karolinska Institutet, S-14186 Stockholm; ${ }^{2}$ Department of Woman and Child Health, Division of Obstetrics and Gynecology, Karolinska University Hospital Solna, Karolinska Institutet, S-17178 Stockholm, Sweden; ${ }^{3}$ The Water and Salt Research Center, Institute of Anatomy, and Institute of Experimental Clinical Research, University of Aarhus, Denmark
\end{abstract}

Received August 23, 2006; Accepted September 29, 2006

\begin{abstract}
Aquaporin-1 (AQP1) is a water channel protein expressed in vascular endothelia and involved in impaired angiogenesis in tumors. Since angiogenesis is an essential component of the regeneration of the endometrium, we sought to analyze the expression of AQP1 in endometrial blood vessels in normal cyclic endometrium as well as in endometrial biopsies of menorrhagia patients. Endometrial biopsies from 16 patients with menorrhagia and 21 healthy fertile women were used for immunohistochemistry assessment of AQP1-stained endothelial structures. RT-PCR was used to confirm the presence of AQP1 mRNA. We detected the expression of AQP1 solely in endometrial blood vessels in the control group, as well as in menorrhagic endometrium. There was no difference between proliferative and secretory endometrium. Furthermore, we observed that the vascular expression of AQP1 in endometrial blood vessels in the menorrhagia group was significantly lower than in controls $(\mathrm{p}=0.002)$. There was also a significantly lower number of stained vessels per unit area in the menorrhagia group than in the controls $(\mathrm{p}=0.006)$. Thus, the deregulation of aquaporin-1 in menorrhagia may be involved in abnormal endometrial vascular growth and permeability.
\end{abstract}

\section{Introduction}

Excessive menstrual bleeding, menorrhagia (i.e. $>80 \mathrm{ml}$ loss of blood), is a common gynecological problem in women of reproductive age, accounting for $>20 \%$ of outpatient clinic

Correspondence to: Dr Sonia Andersson or Dr Miriam Mints, Department of Obstetrics and Gynecology, Karolinska University Hospital Huddinge, Karolinska Institutet, S-14186 Stockholm, Sweden

E-mail: sonia.andersson@telia.com; miriam.mints@telia.com

Key words: angiogenesis, endometrium, menorrhagia, aquaporin-1 visits (1). This disorder may not just cause iron deficiency anemia and require hysterectomy, but may also cause considerable social discomfort and reduced quality of life (2).

Approximately $50 \%$ of menorrhagia patients present no evidence of uterine pathology, suggesting a defect in the cellular processes and regulatory mechanisms of menstruation. An emerging concept suggests that disorders in angiogenesis may be a feature of abnormal or extensive menstrual bleeding (3).

The Aquaporin (AQPs) family comprises at least ten homologous water-transporting proteins in mammals. They are expressed in epithelial, endothelial, and other tissues (4). Structural studies of AQPs indicate a homotetrameric assembly in membranes in which each monomer contains six tilted segments that form a barrel surrounding a water pore (5). Functional measurements indicate that AQPs 1, 2, 4, 5 and 8 are primarily water selective, whereas aquaporins $3,7,9$, and 10 also transport glycerol and possibly other small solutes (6).

AQP1 protein was expressed strongly in proliferating microvessels in human and rat malign brain tumors, bone marrow microvessels in multiple myeloma and proliferating microvessels in chick embryo chorioallantoic membrane (7). Previous studies supported a role for AQP1 in microvessel formation and function. Saadoun et al showed that tumor growth was greatly slowed and microvascular density was lower in AQP1-deficient mice (8). Furthermore, cell migration was greatly impaired in AQP1-deficient cells with abnormal vessel formation in vitro (9).

Since angiogenesis is an essential component of the regeneration of the endometrium, we sought to analyze the hypothesis that menorrhagia arises because of aberrant expression and distribution of AQP1 in the menorrhagic endometrium.

\section{Materials and methods}

Study material. Endometrial biopsies were obtained from 16 normal ovulating women (mean age 42 years) with a history 
of menorrhagia and 21 normal healthy ovulating agematched women (mean age 38 years). Biopsies were obtained using a thin plastic catheter for endometrium samples (Pipelle Laboratoire C.C.D., Paris, France). All women were non-smokers and had not used drugs, hormonal or intrauterine contraception for at least three months prior to the biopsy sampling. Preoperative blood samples showed normal values for platelets, activated prothrombin thromboplastin time, INR, bleeding time and von Willebrand factor. In the women with menorrhagia the uterine cavity was evaluated by hysteroscopy. These women had no discernible endometrial pathology detected by this procedure or routine histological examination.

The stage of the menstrual cycle was based on the date of the last menstruation, analyses of estradiol and progesterone and the histological pattern of the biopsies. Seven women with menorrhagia and 10 women from the control group were in the proliferative phase, and nine women with menorrhagia and 11 from the control group were in the secretory phase.

The study was approved by the Ethics Committee of the Karolinska Hospital. All women gave informed consent to the study.

Immunohistochemistry. Biopsies were fixed in a neutral buffered $4 \%$ formaldehyde solution. After fixation, dehydration and embedding in paraffin, serial sections were cut to a normal thickness of $5 \mu \mathrm{m}$. Thereafter, the sections were deparaffinized in Bioclear (Bio-Optica, Milan, Italy), rehydrated in decreasing concentrations of ethanol $(99.5 \%$, $95 \%$ and $70 \%$ ), and washed in water. After washing the sections in PBS, non-specific antibody binding was reduced by incubating the sections with normal goat serum $(1.5 \%$, Vector Laboratories Inc., Burlingame, CA, USA) in PBS. The sections were then incubated with polyclonal rabbit antigoat AQP1 antibody overnight at $4^{\circ} \mathrm{C}(10)$.

The next day the sections were rinsed in PBS and thereafter incubated with the goat anti-rabbit antibody (Vector Laboratories Inc.). After rinsing in PBS containing $0.01 \%$ Tween-20, staining procedures were performed with a standard avidin-biotin complex (ABC) method (Vectastain ABC Elite, Vector Laboratories Inc.). As negative control, the primary antibody was replaced by normal goat serum. The antigen-antibody reaction was visualized using chromogen 3,3'-diaminobenzidine (DAB-kit, Vector Laboratories Inc.). The slides were counterstained with Harris' hematoxylin.

After coding the slides, they were examined by two independent observers. Examination was conducted using a light field microscope. An average value from the two observers was calculated.

The staining intensity of AQP1 and the number of stained vessels were analyzed in six to ten random fields, depending on the sizes of the samples, using x400 ocular magnification. The staining was graded as follows: $0=$ no detectable staining,$+=$ weak staining pattern,$++=$ moderate staining pattern, and $+++=$ marked (strong) staining pattern. The number of blood vessels was given as the number of profiles per area.

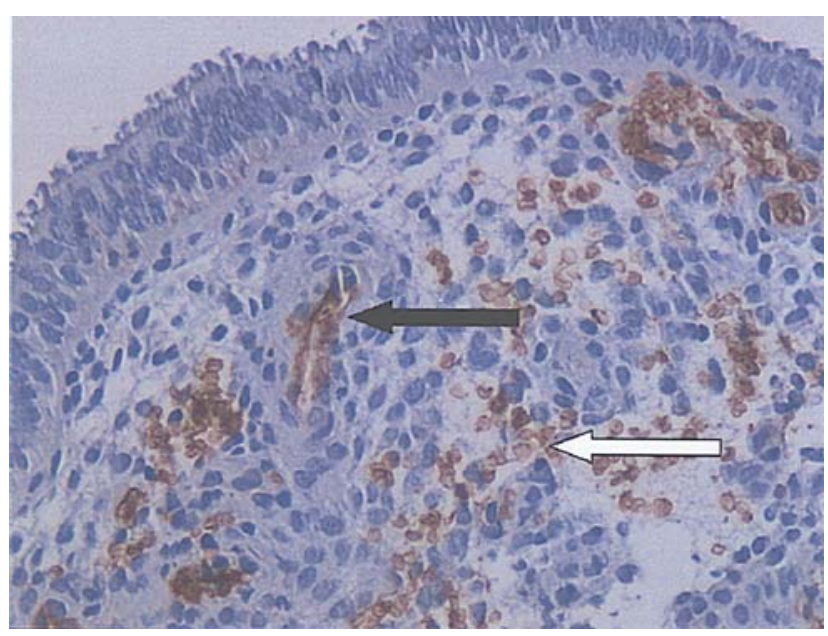

Figure 1. Immunohistochemical staining of AQP1 expression in the endometrium. Intense staining was observed in blood vessels (black arrow) and erythrocytes (white arrow). No staining was observed seen in stroma and epithelial cells.

$P C R$. Total tissue RNA was isolated using the SV Total RNA Isolation System (Promega Corp., Madison, WI, USA) according to the manufacturer's protocol. The extracted RNA was treated with DNase, and its quality was checked on an agarose gel. Total RNA ( $2 \mu \mathrm{g})$ was reverse-transcribed using the First-Strand cDNA Synthesis kit (Pharmacia Biotech AB, Uppsala, Sweden). Then $2 \mu \mathrm{g}$ cDNA was amplified in a total volume of $25 \mu 1$, composed of 10X PCR buffer $(100 \mathrm{mM}$ Tris- $\mathrm{HCl}, \mathrm{pH} 8.4$, and $500 \mathrm{mM} \mathrm{KCl}), 0.5 \mu \mathrm{ldNTP}, 2 \mu \mathrm{l}$ of each primer $(4 \mu \mathrm{M}), 0.15 \mu 1$ TaqDNA polymerase, and $15.85 \mu 1$ sterile water. AQP2 specific primers were generated from known sequences; the forward primer sequence was GTCT TCATCAGCATCGGTTC and the reverse, GTCGGCATC CAGGTCATACT.

The amplification cycle included denaturation at $95^{\circ} \mathrm{C}$ for $3 \mathrm{~min}, 25$ cycles of amplification at $94^{\circ} \mathrm{C}$ for $30 \mathrm{sec}$, annealing at $64^{\circ} \mathrm{C}$ for $45 \mathrm{sec}$, and extension at $72^{\circ} \mathrm{C}$ for $1 \mathrm{~min}$.

Visualization of RT-PCR products was conducted with electrophoresis using a $1.5 \%$ agarose gel with a subsequent treatment with ethidium bromide. As a standard, a 100-base pair ladder (Pharmacia Biotech AB) was chosen.

Statistical analysis. The data did not consistently fulfill the assumptions necessary for using the analysis of variance and t-test; therefore, the non-parametric Kruskal-Wallis and Mann-Whitney U tests were used. Statistical analyses were performed with the Sigma Stat software package (Systat Software GmbH, Erkrath, Germany). p $<0.05$ was considered significant.

\section{Results}

AQP1 expression was detected solely in endometrial blood vessels. Endometrial glands and stroma stained completely negative for AQP1 (Fig. 1).

Moreover, when the menstrual phases were compared we noted that there was no difference in the staining intensity between the proliferative and secretory phase endometrium (Table I). 
Table I. Staining intensity in endometrial vessels.

\begin{tabular}{lllrr}
\hline & \multicolumn{1}{c}{ Sample } & No. of biopsies & $\begin{array}{c}\text { Number of stained vessels } \\
\text { average (SD) }\end{array}$ & $\begin{array}{c}\text { Staining intensity } \\
\text { median (range) }\end{array}$ \\
\hline Control & Proliferative phase & $\mathrm{n}=10$ & $11.23(7.99)^{\mathrm{a}}$ & $2.18(1.99-2.64)^{\mathrm{a}}$ \\
& Secretory phase & $\mathrm{n}=11$ & $13.77(11.55)^{\mathrm{a}}$ & $2.19(1.3-3.00)$ \\
Menorrhagia & Proliferative phase & $\mathrm{n}=7$ & $3.71(3.82)^{\mathrm{b}}$ & $1.84(0.00-2.41)$ \\
& Secretory phase & $\mathrm{n}=9$ & $5.25(3.47)^{\mathrm{b}}$ & $1.3(0.00-2.13)$ \\
\hline
\end{tabular}

Statistical analysis was performed according to the Kruskal-Wallis test; ${ }^{a}$ is statistically separated from ${ }^{\mathrm{b}}, \mathrm{p}<0.05$.
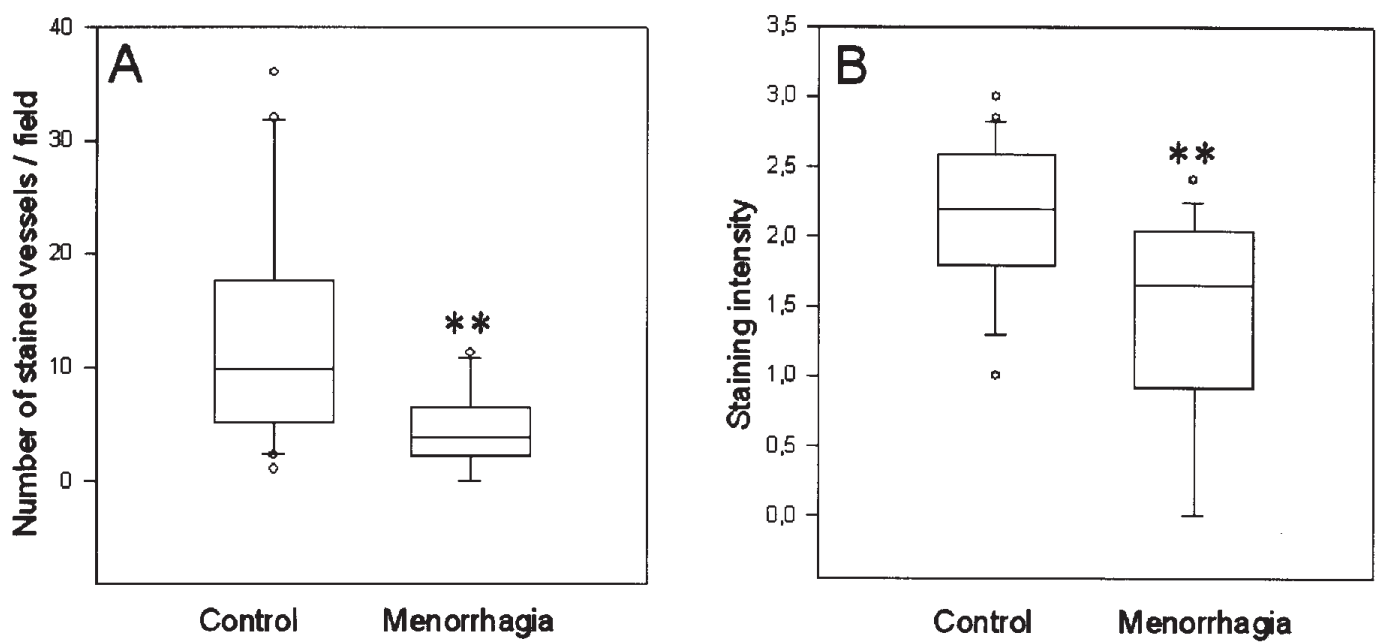

Figure 2. AQP1 staining in the endometrium from control women and women with menorrhagia. (A) Average number of stained vessels/field and (B) staining intensity. Statistical analysis was performed according to the Mann-Whitney U test, $\mathrm{p}<0.01$. o, outliers indicate the specific value outside the box; **statistical significance.

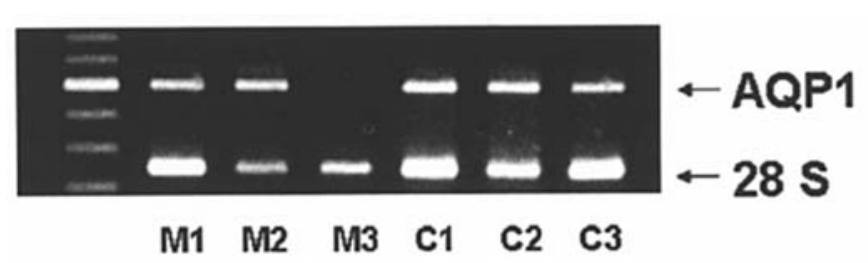

Figure 3. Expression of AQP1 in the endometrium collected from menorrhagia (M1-M3) and control subjects (C1-C3). In one of the samples from the menorrhagia patients (M3), the RT-PCR failed to detect AQP1.

Furthermore, when we assessed the vascular expression of AQP1 as the number of blood vessels staining for this protein per area, we observed significantly more stained vessels in the control group than in the menorrhagia group ( $\mathrm{p}=0.006$ ) (Fig. 2). However, the number of AQP1-positive vessels did not differ during the menstrual cycle between the control group and menorrhagia patients.

We noted also that the menorrhagia group showed significantly lower staining intensity of AQP1 compared to controls ( $\mathrm{p}=0.002)$ (Fig. 2).

The PCR data confirms the presence of AQP1 in the endometrium (Fig. 3).

\section{Discussion}

In this study, we examined the distribution and modulation of AQP1 in normal as well as in menorrhagic endometrium, employing immunohistochemistry and PCR technique.

Biopsies were collected from consecutive patients with idiopathic menorrhagia, selected further to avoid confounding factors, such as smoking, use of contraceptive pills and abnormal estrogen blood levels; since it is well known that cigarette smoking has an anti-estrogenic effect, and sex hormones influence the endometrial regrowth after menstruation.

We demonstrated here that AQP1 was expressed solely in endometrial blood vessels, whereas endometrial glands and stroma stained completely negative for AQP1. Moreover, we found that the number of blood vessels staining for this protein per area was significantly higher in the control group than in the menorrhagia group $(\mathrm{p}=0.006)$. Furthermore, the menorrhagia group showed a significantly lower staining intensity of AQP1 compared to controls $(\mathrm{p}=0.002)$.

Our findings have three important implications. First, human endometrium expresses AQP1. Second, in the functional layer, only the endometrial vessels express AQP1. Third, expression of AQP1 is decreased in menorrhagic endometrium. 
Previously studies indicated the role of AQP1 in angiogenesis. Endo et al (11) and Vacca et al (12) reported strong AQP1 protein expression in tumor microvessels. Using wound closure experiments, Lauffenburger et al showed that cell migration was remarkably enhanced when AQP1 was expressed in the cell, whereas growth and adhesion were not affected by AQP1 expression (9).

Angiogenesis is an essential component of endometrial renewal; however, the timing and the mechanism of new vessel formation in the endometrium during the menstrual cycle are still largely unknown. Using the chick embryo chorioallantoic membrane as an in vivo assay for angiogenesis, Maas et al demonstrated that the endometrium has angiogenic potential throughout the menstrual cycle (13). During the menstrual phase, repair of ruptured blood vessels occurs. Thereafter, during the proliferative phase, the endometrium grows rapidly, supported by the formation of new blood vessels. The secretory phase is characterized by differentiation, which includes the formation of spiral arteries and sub-epithelial capillary plexus (14-16). Two peaks of endothelial cell migratory activity are found in the early and $\mathrm{mid} /$ late proliferative phases of the cycle (17). However, there is no variation in vessel density throughout the menstrual cycle in women with normal blood loss or menorrhagia $(18,19)$.

In this study, we found no difference in AQP1 expression in the proliferative compared to the secretory phase endometrium. There are few studies on the hormonal regulation of AQP1. Results of these studies are contradictory; one study suggested the role of estrogen in AQP1 expression in rat and monkey testes, while a second study did not demonstrate this effect $(20,21)$. Richard et al reported an increased expression of AQP1 in the inner cellular layer of the myometrium in the mouse uterus by exposure to estrogen (22).

Some studies investigated a role of AQP1 in disorders that were associated with endothelial cell dysfunction. The possible role of AQP1 in tumor angiogenesis was investigated by Saadoun et al (8). Melanoma cells were implanted subcutaneously in wild-type and AQP1 null mice. Tumor growth was greatly slowed in the AQP1-deficient mice. Another study reported a decreased expression of AQP1 in human and mouse corneal endothelial dysfunction (Fuchs' dystrophy and bullous keratopathy) (23).

We found that the number of blood vessels stained for AQP1 was significantly lower in the menorrhagia group compared with those of controls. The reason for this changed expression is not yet known. Moreover, the expression of AQP1 in menorrhagia has not been described previously.

It has been suggested that menorrhagia is associated with aberrant angiogenesis. In 1996, Kooy et al observed an increase in endothelial cell proliferation in the endometrium in patients with menorrhagia, compared to the endometrium in the controls (24). Another study demonstrated that the proliferative index of endothelial cells is higher in women with excessive menstrual blood loss (25). Furthermore, our previous study showed vascular abnormalities in the endometrium in menorrhagia (Mints et al, in press). One might speculate that the low levels of AQP1 seen in this study are an expression for changed transendothelial transportation systems relating to permeability changes.
In conclusion, the presence of AQP1 in the endometrial blood vessels indicates the potential involvement of AQP1 in the regulation of angiogenesis in human endometrium. Moreover, aberrant angiogenesis in menorrhagia might be the result of a potential role of AQP1 in the endometrium. Further studies are needed to test whether AQP1-dependent angiogenesis is essential for regeneration of the endometrium.

\section{Acknowledgements}

This study was supported by grants from the Swedish Medical Research Council (nos. 19X-05991, 71XS-131352005-7293), the Karolinska Institutet, Huddinge University Hospital, Swedish Labour Market Insurance and Swedish Society of Medicine, Åke Wiberg Foundation.

\section{References}

1. Hallberg L, Hogdahl AM, Nilsson L and Rybo G: Menstrual blood loss - a population study. Variation at different ages and attempts to define normality. Acta Obstet Gynecol Scand 45: 320-351, 1966.

2. Gath D, Osborn M, Bungay G, Iles S, Day A, Bond A, et al: Psychiatric disorder and gynaecological symptoms in middle aged women: a community survey. Br Med J (Clin Res Ed) 294: 213-218, 1987.

3. Smith SK: Angiogenesis and reproduction. Br J Obstet Gynaecol 108: 777-783, 2001.

4. Verkman AS: Aquaporin water channels and endothelial cell function. J Anat 200: 617-627, 2002.

5. Agre P, King LS, Yasui M, Guggino WB, Ottersen OP, Fujiyoshi Y, et al: Aquaporin water channels-from atomic structure to clinical medicine. J Physiol 542: 3-16, 2002.

6. Fujiyoshi Y, Mitsuoka K, de Groot BL, Philippsen A, Grubmuller H, Agre P, et al: Structure and function of water channels. Curr Opin Struct Biol 12: 509-515, 2002.

7. Xiang Y, Ma B, Li T, Gao JW, Yu HM and Li XJ: Acetazolamide inhibits aquaporin-1 protein expression and angiogenesis. Acta Pharmacol Sin 25: 812-816, 2004.

8. Saadoun S, Papadopoulos MC, Hara-Chikuma M and Verkman AS: Impairment of angiogenesis and cell migration by targeted aquaporin-1 gene disruption. Nature 434: 786-792, 2005

9. Lauffenburger DA and Horwitz AF: Cell migration: a physically integrated molecular process. Cell 84: 359-369, 1996.

10. Smith BL, Agre P: Erythrocyte Mr 28,000 transmembrane protein exists as a multisubunit oligomer similar to channel proteins. J Biol Chem 266: 6407-6415, 1991.

11. Endo M, Jain RK, Witwer B and Brown D: Water channel (aquaporin 1) expression and distribution in mammary carcinomas and glioblastomas. Microvasc Res 58: 89-98, 1999.

12. Vacca A, Frigeri A, Ribatti D, Nicchia GP, Nico B, Ria R, et al: Microvessel overexpression of aquaporin 1 parallels bone marrow angiogenesis in patients with active multiple myeloma. Br J Haematol 113: 415-421, 2001

13. Maas JW, Groothuis PG, Dunselman GA, de Goeij AF, Struyker Boudier HA and Evers JL: Endometrial angiogenesis throughout the human menstrual cycle. Hum Reprod 16: 1557-1561, 2001.

14. Gargett CE and Rogers PA: Human endometrial angiogenesis. Reproduction 121: 181-186, 2001.

15. Gambino LS, Wreford NG, Bertram JF, Dockery P, Lederman F and Rogers PA: Angiogenesis occurs by vessel elongation in proliferative phase human endometrium. Hum Reprod 17: 1199-1206, 2002

16. Girling JE and Rogers PA: Recent advances in endometrial angiogenesis research. Angiogenesis 8: 89-99, 2005.

17. Rogers PA, Abberton KM and Susil B: Endothelial cell migratory signal produced by human endometrium during the menstrual cycle. Hum Reprod 7: 1061-1066, 1992.

18. Rees MC, Dunnill MS, Anderson AB and Turnbull AC: Quantitative uterine histology during the menstrual cycle in relation to measured menstrual blood loss. Br J Obstet Gynaecol 91: 662-666, 1984. 
19. Mints M, Blomgren B, Falconer C, Fianu-Jonasson A and Palmblad J: Microvascular density, vascular endothelial growth factor A, and its receptors in endometrial blood vessels in patients with menorrhagia. Fertil Steril 84: 692-700, 2005.

20. Fisher JS, Turner KJ, Fraser HM, Saunders PT, Brown D and Sharpe RM: Immunoexpression of aquaporin-1 in the efferent ducts of the rat and marmoset monkey during development, its modulation by estrogens, and its possible role in fluid resorption. Endocrinology 139: 3935-3945, 1998.

21. Oliveira CA, Carnes K, Franca LR, Hermo L and Hess RA: Aquaporin-1 and -9 are differentially regulated by oestrogen in the efferent ductile epithelium and initial segment of the epididymis. Biol Cell 97: 385-395, 2005.

22. Richard C, Gao J, Brown N and Reese J: Aquaporin water channel genes are differentially expressed and regulated by ovarian steroids during the periimplantation period in the mouse. Endocrinology 144: 1533-1541, 2003.
23. Macnamara E, Sams GW, Smith K, Ambati J, Singh N and Ambati BK: Aquaporin-1 expression is decreased in human and mouse corneal endothelial dysfunction. Mol Vis 10: 51-56, 2004.

24. Kooy J, Taylor NH, Healy DL and Rogers PA: Endothelial cell proliferation in the endometrium of women with menorrhagia and in women following endometrial ablation. Hum Reprod 11: 1067-1072, 1996

25. Abberton KM, Taylor NH, Healy DL and Rogers PA: Vascular smooth muscle alpha-actin distribution around endometrial arterioles during the menstrual cycle: increased expression during the perimenopause and lack of correlation with menorrhagia. Hum Reprod 11: 204-211, 1996. 